\title{
Qualidade de vida em mulheres com Incontinência Urinária
}

\author{
Quality of life in women with Urinary Incontinence \\ Calidad de vida en mujeres con Incontinencia Urinaria
}

\author{
Susana Fernandes*; Emília Carvalho Coutinho**; João Carvalho Duarte***; \\ Paula Alexandra Batista Nelas ${ }^{* * *}$; Claudia Margarida Correia Balula Chaves*****; \\ Odete Amaral ${ }^{* * * * * *}$
}

\section{Resumo}

Enquadramento: A Incontinência Urinária (IU) provoca alterações na Qualidade de Vida (QV) da mulher, com limitações fisiológicas, psicológicas e socioeconómicas.

Objetivos: Avaliar a perceção da QV das mulheres com IU; analisar a influência do tipo de IU na QV; analisar a influência das variáveis sociodemográficas, obstétricas e ginecológicas na QV.

Metodologia: Estudo transversal e analítico. Os dados foram recolhidos por questionário constituído por variáveis sociodemográficas, obstétricas e ginecológicas e o King's Health Questionnaire. Amostra com 305 mulheres, da região centro de

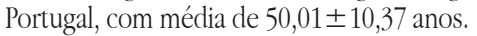

Resultados: As mulheres revelaram moderada qualidade de vida global, sendo que na generalidade das dimensões, esta é influenciada pela idade, nacionalidade, situação profissional atual, área de residência, índice de massa corporal, número de filhos, lesões pélvicas no trabalho de parto, peso da criança à nascença, anos de perda de urina, incontinência urinária de esforço, incontinência urinária de urgência. As mulheres com IU de esforço apresentam pior qualidade de vida.

Conclusão: A incontinência urinária influencia moderadamente a QV da mulher.

Palavras-chave: incontinência urinária; mulher; qualidade de vida.

\begin{abstract}
Background: Urinary Incontinence (UI) impacts the Quality of Life (QoL) of women, causing physiological, psychological, and socioeconomic limitations.

Objectives: To assess the perception of QoL of women with UI; to analyse the influence of the type of UI on QoL; to analyse the influence of socio-demographic, obstetric, and gynaecological variables on QoL. Methodology: A cross-sectional analytic study was conducted. Data were collected using a questionnaire consisting of socio-demographic, obstetric, and gynaecological variables, and the King's Health Questionnaire. The sample was composed of 305 women from the central region of Portugal, with a mean age of $50.01 \pm 10.37$ years. Results: Overall, women showed moderate QoL. In most dimensions, QoL is influenced by age; nationality; current employment status; area of residence; bodv mass index; number of children; pelvic lesions during delivery; child's weight at birth; years of urine loss; stress incontinence; urge incontinence. Women with urinary stress incontinence have a worse QoL.

Conclusion: UI moderately affects the QoL of women.
\end{abstract}

Keywords: urinary incontinence; women; quality of life.

Mestre em Saúde Materna, Obstetrícia e Ginecologia. Enfermeira Especialista. Unidade de Cuidados pt]. Contribuição no artigo: pesquisa bibliográfica, recolha de dados, tratamento dos dadōs, análise e discussão dos dados, escrita do artigo

** Doutora em Ciências de Enfermagem. Professora Adjunta, Instituto Politécnico de Viseu, 3500-843, Viseu, Portugal [ecoutinhoessv@gmail.com]. Contribuição no artigo: pesquisa bibliográfica, recolha de dados, tratamento dos dados, análise e discussão dos dados, escrita do artigo, revisão do conteúdo *** Doutor em Saúde Mental. Professor Coordenador, Instituto Politécnico de Viseu, 3500-843. Viseu, Portugal [Duarte.johnny@gmail.com]. Contribuição no artigo: tratamento, análise e discussão dos dados, revisão do conteúdo

****Doutora em Ciências da Educação. Professora Adjunta, Instituto Politécnico de Viseu, 3500-843, Viseu, Portugal [pnelas@gmail.com]. Contribuição no artigo: pesquisa bibliográfica, discussão dos dados, escrita do artigo, revisão do conteúdo

****** Doutora em Ciências da Educação. Professora Adjunta, (. Instituto Politécnico de Viseu, 3500-843, Viseu, Portugal [claudiachaves21@gmail.com]. Morada para correspondência: Rua da Fonte, $\mathrm{n}^{\circ} .12$ Outeiro da Comenda, São Pedro do Sul, 3500-843, Viseu, Portugal. Contribuição no artigo: pesquisa bibliográfica, discussão dos dados, escrita do artigo, revisão do conteúdo

******* Doutora em Saúde Pública. Professora Adjunta, Instituto Politécnico de Viseu, 3500-843, Viseu, Portugal [mopamaral@gmail.com]. Contribuição no artigo: pesquisa bibliográfica, tratamento dos dados, análise e discussão dos dados, revisão do conteúdo

\section{Resumen}

Marco contextual: La Incontinencia Urinaria (IU) origina alteraciones en la Calidad De Vida (CDV) de la mujer, con limitaciones fisiológicas, psicológicas y socioeconómicas.

Objetivos: Evaluar la percepción de la CDV de las mujeres con IU; analizar la influencia del tipo de IU en la CDV; analizar la influencia de las variables sociodemográficas, obstétricas y ginecológicas en la CDV.

Metodología: Estudio transversal y analítico. Los datos se recogieron a través de un cuestionario constituido por variables sociodemográficas, obstétricas, ginecológicas y la escala King's Health Questionnaire. La muestra estuvo formada por 305 mujeres de la región centro de Portugal con una edad media de 50,01 $\pm 10,37$ años.

Resultados: Las mujeres mostraron una CDV moderada y que esta, en la mayoría de las dimensiones, está influida por la edad, la nacionalidad, la situación profesional actual, el área de residencia, el índice de masa corporal, el número de hijos, las lesiones pélvicas durante el parto, el peso del niño al nacer, los años de pérdidas de orina, la IU de esfuerzo (IUE) y la IU de urgencia. Las mujeres con IUE presentan peor CDV.

Conclusión: La incontinencia urinaria afecta moderadamente a la CDV de la mujer.

Palabras clave: incontinencia urinaria; mujer; calidad de vida.

Recebido para publicação em: 04.06 .14

Aceite para publicação em: 15.12 .14 


\section{Introdução}

A Incontinência Urinária (IU) é uma patologia conhecida pelo impacto negativo que causa em diferentes domínios da vida da mulher, não só a nível físico mas também a nível psíquico, emocional e social, com custos substanciais a nível económico (Botlero, Urquhart, Davis, \& Bell, 2008). Pode afetar mulheres de todas as idades, influenciar a saúde uroginecológica e a Qualidade de Vida (QV) da mulher e família (Correia, Dinis, Rolo, \& Lunet, 2009). Em Portugal e, de acordo com a Associação Portuguesa de Urologia, estima-se que existam 600 mil pessoas incontinentes nos diferentes grupos etários, entre os 45 e os 65 anos de idade, sendo a proporção de casos de incontinência urinária de três mulheres para cada homem. Cerca de $50 \%$ das pessoas institucionalizadas sofrem de incontinência urinária e apenas 10\% da população faz tratamento farmacológico, sendo a taxa de cura da incontinência de esforço de 90\% (Associação Portuguesa de Urologia [APU], 2010). Esta problemática é muitas vezes negligenciada ao nível da saúde uroginecológica da mulher, tanto pelas próprias, quanto pelos profissionais de saúde, quer por desconhecimento ou por falta de sensibilidade, negligenciando-se a sua verdadeira extensão e impacto. As mulheres com IU ficam frequentemente afetadas pela sua condição física e tendem a limitar as suas atividades de âmbito social, não tendo consciencialização de que se trata de uma patologia, de um modo geral tratável, sendo que no seu imaginário, esta situação está muitas vezes associada ao próprio processo natural de envelhecimento. Por estar estritamente relacionada com a vida íntima da mulher, muitas vezes assiste-se a uma certa relutância em abordar este assunto, ou procurar ajuda para a resolução do problema, e estas mulheres acabam por se isolar, familiar e socialmente, com implicações agravadas pelo adiamento ou ausência de tratamento. Pelo exposto, o presente estudo tem como objetivos avaliar a perceção da QV das mulheres com IU; analisar a influência do tipo de IU na QV e analisar a influência das variáveis sociodemográficas, obstétricas e ginecológicas na QV.

\section{Enquadramento}

A incontinência urinária, ou perda involuntária de urina é uma patologia que afeta sobretudo as mulheres e pode ser classificada de esforço - quando ocorre após esforço, tosse ou espirro; de urgência quando a vontade de urinar é súbita e incontrolável; e mista - quando existem sinais e sintomas dos dois tipos mencionados (Botelho, Silva, \& Cruz, 2007).

É reconhecida como um problema orgânico objetivo, de etiologia multifatorial, e com consequências nefastas a nível físico e bem estar (higiene, mau odor e vestuário molhado); psicológico (diminuição da autoestima e autoconceito, aumento dos níveis de stresse, humor depressivo ou depressão); sociocultural (isolamento social e diminuição das atividades relacionais), profissional (absentismo laboral, menor produtividade ou mesmo alteração radical dos ritmos de trabalho); e económicofinanceiro (despesa acrescida em roupa interior, fraldas, entre outros). Estas consequências conduzem a uma pior qualidade de vida das mulheres nos diferentes grupos etários (Lasserre et al., 2009; Basak, Kok, \& Guvenc, 2013).

Apesar das perdas involuntárias de urina interferirem de forma devastadora na qualidade de vida das mulheres, a incontinência urinária feminina continua a ser sub-diagnosticada e sub-tratada. Estima-se que apenas uma em cada quatro mulheres sintomáticas procura ajuda médica, já que é considerada de forma errónea uma consequência natural da idade e sem tratamento eficaz, sendo por isso uma epidemia silenciosa (Botelho, Silva, \& Cruz, 2007). As principais causas da incontinência urinária são a perturbação cerebral, a infeção, as alterações das vias do sistema nervoso central, as alterações do reflexo uretrovesical e as lesões nos tecidos. Os fatores que estão afetados são a consciência da necessidade de urinar, a capacidade cortical para inibir a micção, e a reação do cérebro ao enchimento da bexiga (Weigel \& Potter, 2010).

As evidências sobre a importância da qualidade de vida dos indivíduos têm vindo a aumentar de interesse. Este é um construto aplicado a vários níveis, da medicina à saúde pública, da sociologia à economia e da política à psicologia. Contudo, permanece uma falta de consensualidade na definição do conceito (Pais-Ribeiro, 2009). O conceito de QV da Organização Mundial da Saúde (OMS) inclui uma perspetiva cross-cultural, sendo esta descrita como uma perceção individual sobre a própria posição na vida num determinado contexto cultural e sistema de valores nos quais o indivíduo vive, e em relação aos 
seus objetivos, expectativas, metas e preocupações/ interesses. Este conceito positivo de saúde enfatiza os recursos pessoais e sociais, assim como as capacidades físicas do indivíduo (Pais-Ribeiro, 2009). Dado que a saúde surge como um domínio importante da QV é fulcral considerar a gravidade da IU em relação à sua sintomatologia, ao seu impacto psicológico e social e à forma como afeta as atividades de vida diária, as atividades sociais, as relações e as emoções de uma forma geral. Estudos têm referido que os diferentes tipos de IU afetam de forma distinta a QV da mulher. As mulheres com IU de urgência apresentam pior QV, atribuível à sintomatologia urinária, quando comparadas com as que apresentam IU de esforço (Kelleher, Cardozo, Khullar, \& Salvatore, 1997).

Um estudo realizado por Grimby, Milsom, Molander, Wiklund, e Ekelund (1993), evidenciou níveis significativamente mais elevados de dificuldades emocionais e de isolamento social em mulheres com IU de urgência e mista do que as que sofrem de IU de esforço. De igual modo, as mulheres com IU de urgência apresentam mais distúrbios do sono o que contribui para uma diminuição da QV. Outro estudo que comparou o impacto do tipo de IU sobre a QV em mulheres que realizaram tratamento fisioterapêutico revelou que a maioria apresentava incontinência urinária mista, tendo este tipo de incontinência um impacto negativo significativamente maior sobre a QV, sobretudo no domínio da perceção geral da saúde em comparação com as doentes com incontinência urinária de esforço (Dedicação, Haddad, Saldanha, \& Driusso 2009). Os sintomas que mais afetam a QV das mulheres estão associados à urgência miccional, à IU de esforço, à frequência urinária e à incontinência de urgência (Knorst, Resende, \& Goldim, 2010).

Investigações indicaram que existem vários fatores associados com a qualidade de vida das mulheres com incontinência, designadamente fatores do foro ginecológico, obstétrico e sociodemográfico (Botlero, Davis, Urquhartb, Shortreed, \& Bell, 2009; Correia et al., 2009; Basak et al., 2013; Sensoy, Dogan, Ozek, \& Karaaslan, 2013; Kirss, Lang, Toompere, \& Veerus, 2013).

Pelo exposto, a IU é uma condição que afeta de forma negativa a QV das mulheres no seu contexto biopsicossocial pelas limitações impostas pela patologia. Assim, urge a necessidade de reunir esforços para sensibilizar os profissionais de saúde para a adoção de medidas de saúde uroginecológicas que previnam ou tratem precocemente a IU na população feminina.

\section{Questóes de Investigaçáo}

Perante esta problemática, pretende-se responder às seguintes questões de investigação: Qual a perceção da qualidade de vida das mulheres com incontinência urinária? Qual a influência do tipo de incontinência urinária na qualidade de vida? Quais as variáveis que influenciam a qualidade de vida das mulheres com incontinência urinária?

\section{Metodologia}

Realizou-se um estudo transversal analítico. Recorreu-se a uma amostragem não probabilística por conveniência, constituída por mulheres com incontinência urinária, utentes de um Centro de Saúde e de um Hospital da região centro de Portugal. Os dados foram recolhidos entre 2011 e 2012. O estudo teve autorização do Agrupamento de Centros de Saúde e do Hospital, e da Comissão Nacional de Proteção de Dados, da qual se obteve a respetiva autorização (CNPD referência 20.789.050). Consideraram-se os seguintes critérios de inclusão: presença de sintomatologia de incontinência urinária, sem outra patologia associada; capacidade para falar, ler e escrever; e autorização escrita e individual para a participação no estudo depois de concedidas as devidas informações e esclarecimentos relativos ao estudo.

A recolha de dados foi efetuada através de um questionário aplicado face a face, constituído por variáveis sociodemográficas, obstétricas e ginecológicas, e o King's Health Questionnaire para avaliar a qualidade de vida das mulheres com IU. O King's Health Questionnaire encontra-se validado para a população portuguesa (Tamanini, D' Ancona, Botega, \& Rodrigues Netto Jr, 2003) e categorizado em nove domínios: perceção geral da saúde, impacto da incontinência urinária, limitação de atividades diárias, limitação física, limitação social, relações pessoais, emoções, sono/energia e medidas de gravidade, sendo que, a primeira parte do questionário reportase à perceção geral de saúde e ao impacto da incontinência e na segunda parte são explorados seis 
domínios: papel, limitações físicas e sociais, pessoais, relacionamentos, as emoções e o sono. É pontuado por cada um dos seus domínios. As pontuações variam de 0 a 100 e quanto maior a pontuação obtida, pior é a qualidade de vida. Com o objetivo de ilustrar a qualidade de vida estabelecemos os seguintes pontos de corte: $\leq 50$ - qualidade de vida fraca; 51 a 66 qualidade de vida moderada $\mathrm{e} \geq 66$ - qualidade de vida elevada, com base na fórmula de grupos extremos de corte (Pestana \& Gageiro, 2005). Utilizou-se a estatística paramétrica e não paramétrica recorrendose a esta última quando as condições de aplicação da primeira não se verificavam, principalmente quando os tamanhos amostrais não eram homogéneos, isto é, o quociente entre o $\mathrm{N}$ maior sobre o $\mathrm{N}$ menor é superior a 1,5. Como testes paramétricos fizemos uso do teste $t$ de student para amostras independentes, análise de variância a um factor para comparação de médias em mais de dois grupos amostrais e como teste não paramétrico o teste de UMann Wbitney em substituição do teste t e o teste de qui quadrado para variáveis nominais.

No processamento e análise dos dados foi utilizado o programa Statistical Package for the Social Sciences (SPSS 20.0.). A amostra final ficou constituída por 305 mulheres com incontinência urinária, com idades entre os 29 e os 75 anos ( $X=50,01 \pm 10,37$ anos). A maioria das mulheres era de raça branca $(98,0 \%)$, nacionalidade portuguesa (95,4\%), casadas/união de facto $(90,1 \%)$, domésticas $(30,9 \%)$, desempregadas $(54,9 \%)$, a maioria com habilitações literárias o $3^{\circ}$ ciclo de escolaridade $(70,2 \%)$, residentes em vila $(44,7 \%)$ e classifica o seu rendimento mensal entre os 500 e os 1000 euros $(47,7 \%)$.

Tabela 1

Qualidade de vida das mulheres com IU de esforço e de urgência

\begin{tabular}{|c|c|c|c|c|c|c|c|c|}
\hline \multirow{5}{*}{ IU Esforço } & \multicolumn{8}{|c|}{ Qualidade de Vida } \\
\hline & & \multicolumn{2}{|c|}{ Fraca } & \multicolumn{2}{|c|}{ Moderada } & \multicolumn{2}{|c|}{ Elevada } & \multirow[b]{2}{*}{$p$} \\
\hline & & $\mathrm{n}$ & $\%$ & $\mathrm{n}$ & $\%$ & $\mathrm{n}$ & $\%$ & \\
\hline & $\operatorname{sim}$ & 37 & 17,9 & 62 & 30,0 & 108 & 52,2 & 0,001 \\
\hline & não & & & & & & & \\
\hline \multirow{2}{*}{ IU Urgência } & $\operatorname{sim}$ & 35 & 50,0 & 10 & 14,3 & 25 & 35,7 & 0,05 \\
\hline & não & & & & & & & \\
\hline
\end{tabular}

Da análise descritiva das variáveis obstétricas verificamos que as mulheres estiveram grávidas uma

\section{Resultados}

No que concerne à qualidade de vida em função da idade das mulheres inquiridas, verifica-se que 39,5\% apresenta uma qualidade de vida elevada, $38,4 \%$ das mulheres apresenta uma qualidade de vida fraca e 22,1\% uma qualidade de vida moderada. Estratificando por grupos etários, observamos que a maior percentagem das mulheres que referiu uma qualidade de vida fraca e moderada tem uma idade $\leq 45$ anos ( $40,7 \%$ vs. $37,0 \%$ e $27,4 \%$ vs. $18,8 \%$; respetivamente) e a maior percentagem de mulheres que mencionou uma qualidade de vida elevada tem uma idade $\geq 46$ anos ( $44,2 \%$ vs. $31,9 \%$ ); embora as diferenças entre a idade e o total da qualidade de vida não sejam significativas $(p=0,071)$. Contudo, a idade associou-se com algumas dimensões do King's Health Questionnaire, designadamente com a perceção geral da saúde $(p<0,001)$; limitações nas atividades diárias ( $p=0,001)$; limitações físicas $(p=0,001)$ e com a dimensão emoções $(p=0,05)$.

Em relação ao tipo de incontinência, a prevalência de IU de esforço foi de $71,9 \%$ e de IU de urgência de 23,4\%. Na amostra total, 39,5\% das mulheres com IU referiram elevada qualidade de vida, 22,1\% moderada qualidade de vida e $38,4 \%$ fraca qualidade de vida.

Das mulheres com IU de esforço, 17,9\% referiram fraca qualidade de vida; 30,0\% moderada e 52,2\% elevada qualidade de vida, com diferenças estatisticamente significativas $(p<0,001)$. Das mulheres com IU de urgência, 50,0\% referiram fraca qualidade de vida; $14,3 \%$ moderada e $35,7 \%$ elevada qualidade de vida, com diferenças estatisticamente tangenciais ( $p=0,05$; Tabela 1). 
esteve em trabalho de parto no primeiro filho $7 \mathrm{ou}$ mais horas $(39,7 \%)$, no segundo filho 7 ou menos horas $(37,2 \%)$ e no terceiro filho 4 ou menos horas (41,9\%). Verificou-se que $80,0 \%$ das mulheres referiu não ter lesões pélvicas, como lacerações durante os partos. A maioria das mulheres teve filhos com peso superior a $4 \mathrm{Kg}$ (72,5\%). Relativamente às variáveis ginecológicas e analisando o número de anos de perda de urina verificou-se um mínimo de 1 ano e um máximo de 30 anos de perda de urina, a maioria das mulheres perde urina há 5 ou menos anos $(35,1 \%)$ e há 10 ou mais anos (35,1\%). Em relação ao número de infeções vaginais/dermatites que apresentaram durante o último ano foi de 1 a 6 episódios. A maioria das mulheres não tem conhecimentos dos exercícios de Kegel (84,3\%), nunca foi submetida a cirurgias para correção da incontinência urinária (98,0\%); usa pensos absorventes (84,6\%) e não associa o início da perda de urina a algum momento da sua vida $(98,9 \%)$. A qualidade de vida total associou-se com variáveis sociodemográficas - estado civil $(p<0,01)$, situação profissional atual $(p<0,001)$ e rendimento mensal ( $p<0,001)$; com variáveis obstétricas - índice de massa corporal $(p<0,01)$, número de filhos $(p<0,001)$ e peso da criança à nascença $(p<0,001)$; com o tipo de incontinência - IU de esforço ( $p<0,001$; Tabela 2).

Tabela 2

Fatores associados com a qualidade de vida em mulheres com IU

\begin{tabular}{lcc}
\hline & \multicolumn{2}{c}{ Qualidade de Vida Total } \\
& Teste & $p$ \\
\hline Variáveis sociodemográficas & & \\
$\quad$ Estado civil & $\mathrm{U}=2752,50$ & 0,003 \\
$\quad$ Situação profissional atual & $\mathrm{T}=-3,928$ & 0,000 \\
$\quad$ Rendimento mensal & $\mathrm{U}=10,711$ & 0,000 \\
Variáveis Obstétricas & $\mathrm{U}=8154,00$ & 0,005 \\
$\quad$ Índice de massa corporal & $\mathrm{F}=10,291$ & 0,000 \\
$\quad$ Número de filhos $(>2)$ & $\mathrm{U}=177,89$ & 0,002 \\
$\quad$ Peso da criança à nascença (superior a 4Kg) & & \\
Tipo de IU & $\mathrm{U}=1973,50$ & 0,000 \\
$\quad$ IU esforço & &
\end{tabular}

Nota. U - U de Mann-Whitney; T - teste t de student; F - One-way ANOVA

\section{Discussáo}

É extremamente importante avaliar o impacto e a perceção da qualidade de vida em mulheres com IU. Vários estudos têm concluído que as mulheres com IU frequentemente apresentam uma diminuição da sua qualidade de vida (Correia et al., 2009; Lasserre et al., 2009; Basak et al., 2013).

Os dados obtidos no presente estudo estão em consonância com os alcançados por Dedicação et al. (2009), no que se refere à IU causar um importante e substancial impacto negativo na QV das mulheres, ainda que as mesmas tenham percecionado a sua QV geral como moderada. No referido estudo também se evidenciaram as mulheres com IU de esforço com pior qualidade de vida, corroborando também os dados obtidos por Oliveira et al. (2010). Salvaguardase o caráter subjetivo da avaliação da QV, que pode ocorrer da diversidade de fatores sociais, culturais, religiosos e higiénicos que cada participante possui.

A incontinência urinária é um problema relativamente frequente e desagradável. Em relação aos aspetos obstétricos e analisando o estudo de Albuquerque et al. (2011), 65,5\% das mulheres engravidou pelo menos uma vez, o mais frequente foi duas gestações e uma gestação, das quais a maioria foi sujeita a cesariana, o que não corrobora os dados obtidos na presente investigação, na medida em que se verificou que houve uma prevalência de mulheres que teve parto normal.

Constatou-se, ainda, que grande parte das mulheres não tem conhecimento dos exercícios de Kegel. Este tipo de exercícios pela sua simplicidade e reduzido custo devem ser sempre recomendados, não apenas como tratamento inicial das incontinências ligeiras, mas sobretudo como medida preventiva após o 
parto vaginal ou cirurgias sobre a região pélvica (Botelho, Silva, \& Cruz, 2007). Como demonstram os resultados, vários são os fatores de risco associados à IU, estando os mesmos descritos na literatura, como por exemplo a idade, a nacionalidade, o índice de massa corporal, o número de filhos, as lesões pélvicas no trabalho de parto, o peso da criança à nascença, o exercício físico, entre outros; sugerindo que esta é parte de um problema complexo, decorrente da sua natureza multifatorial, em virtude das transformações vasculares e do sistema nervoso central, que afetam os mecanismos de controlo da bexiga, particularmente com o avanço da idade (Albuquerque et al., 2011; Basak et al., 2013; Sensoy et al., 2013).

Os resultados apresentados em relação às variáveis ginecológicas sugerem a necessidade de fazer um rastreio dos sintomas urinários e dos fatores a eles associados, para que possam, o mais precoce possível, ser detetados pelos profissionais da saúde, que devem estar atentos à condição altamente frequente entre as mulheres, possibilitando, assim, uma adequada abordagem preventiva (Dellú, Zácaro, \& Schmitt, 2008). Neste sentido, Dellú et al. (2008) referem que as atividades sociais, familiares e profissionais podem ficar restritas em mulheres com incontinência urinária e diminuir a sua qualidade de vida, ao gerar isolamento social e stresse emocional, associado ou não à sensação de inferioridade e depressão, adicionando-se a tais consequências físicas e sociais a encargos financeiros, que é substancial e crescente.

\section{Conclusáo}

A incontinência urinária tem impacto na vida da maioria das mulheres, ressaltando a incontinência urinária de esforço. Na generalidade, constatou-se que a QV, avaliada pelas suas subescalas, é influenciada pela idade, nacionalidade, situação profissional atual, área de residência, índice de massa corporal, número de filhos, lesões pélvicas no trabalho de parto, peso da criança à nascença, anos de perda de urina, incontinência urinária de esforço e incontinência urinária de urgência. O tipo de parto não interferiu em nenhuma subescala da qualidade de vida.

A incontinência urinária provoca alterações nas várias dimensões da vida de uma mulher, quer pelas limitações fisiológicas impostas pela doença, quer pelo confronto psicológico face à inibição social e familiar, o que determina implicações na QV. A conjugação da metodologia qualitativa com a quantitativa poderá contribuir para o esclarecimento desta problemática ao procurar estudar a experiência/ vivência destefenómeno, privilegiando a interpretação da experiência vivida pelas mulheres, não só para as conhecer melhor, mas também para saber como intervir no sentido das suas necessidades. De igual modo seria necessário perceber se os profissionais de saúde, nomeadamente médicos e enfermeiros, estão a agir de forma a prevenir a incontinência urinária, e se revelarão a preparação e os conhecimentos necessários para ajudar essas mulheres a ultrapassar esta situação de morbilidade.

Considera-se fundamental que o enfermeiro esteja atento a esta problemática e consiga intervir precocemente a fim de ajudar a mulher a prevenir a IU através da realização de exercícios de Kegel e consciencialização da importância da prevenção e intervenção atempada.

\section{Referencias bibliográficas}

Albuquerque, M. T., Micussi, B. C., Soares, E. M. M., Lemos, T. M. A. M., Brito, T. N. S., Silva, J. B., \& Maranhão, T. M. O. (2011). Correlação entre as queixas de incontinência urinária de esforço e o pad test de uma hora em mulheres na pósmenopausa. Revista Brasileira de Ginecologia e Obstetricia, 33(2), 70-74. Recuperado de http://www.scielo.br/pdf/rbgo/ v33n2/v33n2a03

Associação Portuguesa de Urologia. (2010). Semana da incontinência urinária. Recuperado de http://www. apurologia.pt/

Basak, T., Kok, G., \& Guvenc, G. (2013). Prevalence, risk factors and quality of life in Turkish women with urinary incontinence: A synthesis of the literature. International Nursing Review, 60(4), 448-460. doi: 10.1111/inr.12048.

Botelho, F., Silva, C., \& Cruz, F. (2007). Incontinência urinária feminina. Acta Urológica, 2, 79-82.

Botlero R., Urquhart D. M., Davis S. R., \& Bell R. J. (2008). Prevalence and incidence of urinary incontinence in women: Review of the literature and investigation of methodological issues. International Journal of Urology, 15(3), 230-4. doi: 10.1111/j.1442-2042.2007.01976.x.

Botlero, R., Davis, S. R., Urquhartb, D. M., Shortreed, S., \& Bell, R. J. (2009). Age-specific prevalence of, and factors associated with, different types of urinary incontinence in communitydwelling Australian women assessed with a validated questionnaire. Maturitas, 62, 134-139. 
Correia, S., Dinis, P., Rolo, F., \& Lunet, N. (2009). Prevalence, treatment and known risk factors of urinary incontinence and overactive bladder in the noninstitutionalized Portuguese population. International Urogynecology Journal Pelvic Floor Dysfunction, 20, 1481-1489.

Dedicação, A. C., Haddad, M., Saldanha, M. E. S., \& Driusso, P. (2009). Comparação da qualidade de vida nos diferentes tipos de incontinência urinária feminina. Revista Brasileira de Fisioterapia, 13(2), 1-7. Recuperado de: http://www.scielo. br/pdf/rbfis/v13n2/aop012_09.pdf

Dellú, M. C., Zácaro, P. M. D., \& Schmitt, A. C. B. (2008). Prevalência de sintomas urinários e fatores obstétricos associados em mulheres adultas. Revista Brasileira de Fisioterapia, 12(6), 1-8. Recuperado de http://www.scielo.br/pdf/rbfis/v12n6/ aop005.pdf

Grimby A., Milsom I., Molander U., Wiklund I., \& Ekelund P. (1993). The influence of urinary incontinence on the quality of life of elderly women. Age Ageing, 22(2), 82-9.

Kelleher, C. J., Cardozo, L. D., Khullar, V., \& Salvatore, S. (1997). A new questionnaire to assess the quality of life of urinary incontinent women. British Journal of Obstetrics and Gynaecology, 104, 1374-1379.

Kirss, F., Lang, K., Toompere, K., \& Veerus, P. (2013). Prevalence and risk factors of urinary incontinence among Estonian postmenopausal women. Springerplus, 17(2), 524. doi: 10.1186/2193-1801-2-524.

Knorst, M. R., Resende, T. L. \& Goldim, J. R. (2010). Clinical profile, quality of life and depressive symptoms of women with urinary incontince attending a university hospital. Revista Brasileira de Fisioterapia, 15, 2, 12-23.
Lasserre, A., Pelat, C., Guéroult, V., Hanslik, T., Chartier-Kastler, E., Blanchon, T., \& Bloch, J. (2009). Urinary incontinence in French women: Prevalence, risk factors and impact on quality of life. European Urology, 56(1), 177-83. doi: 10.1016/j. eururo.2009.04.006.

Oliveira, E., Losinsky, A. C., Palos, C. C., Ribeiro, D. A. M., Souza, A. M. B., \& Barbosa, C. P. (2010). Influência do índice de massa corporal na incontinência urinária feminina. Revista Brasileira de Ginecologia e Obstetricia,32(9), 454-458.

Pais-Ribeiro, J. (2009). A importância da qualidade de vida para a psicologia da saúde. In J. P. Cruz, S. N. Jesus, \& C. Nunes (Coords.), Bem-estar e qualidade de vida (pp.31-49). Alcochete, Portugal: Textiverso.

Pestana, M. H., \& Gageiro, J. N. (2005). Análise de dados para ciências sociais: A complementaridade do SPSS ( $4^{\mathrm{a}}$ ed.). Lisboa, Portugal: Edições Silabo.

Sensoy, N., Dogan, N., Ozek, B., \& Karaaslan, L. (2013). Urinary incontinence in women: Prevalence rates, risk factors and impact on quality of life. Pakistan Journal of Medical Sciences, 29(3), 818-22.

Tamanini,J.T. N., D’Ancona, C. A. L., Botega, N.J., \& Rodrigues Netto Jr, N. (2003). Validação do "King's Health Questionnaire" para o português em mulheres com incontinência urinária. Revista de Saúde Pública, 37(2), 203-211. Recuperado de http:// www.scielo.br/scielo.php?script $=$ sci_arttext $\&$ pid $=$ S0034$89102003000200007 \& \operatorname{lng}=$ en\&tlng $=$ pt. $\quad 10.1590 /$ S003489102003000200007.

Weigel, K. A., \& Potter, C. K. (2010). Avaliação do sistema renal. In F. D. Monahan, J. K. Sands, M. Neighbors, J. F. Marek, \& C. J. Green (Eds.), Enfermagem médico-cirúrgica: Perspectivas de saúde e doença (8a ed., Vol. 2, pp. 971-1068.). Loures, Portugal: Lusodidacta. 
